\title{
Sensitivity of a Liquid Xenon Detector to Neutrino-Nucleus Coherent Scattering and Neutrino Magnetic Moment from Reactor Neutrinos
}

\author{
Kaixuan Ni *(D), Jianyang Qi, Evan Shockley (D) and Yuehuan Wei \\ Department of Physics, University of California San Diego, La Jolla, CA 92093, USA; jiq019@ucsd.edu (J.Q.); \\ eshockley@physics.ucsd.edu (E.S.); ywei@physics.ucsd.edu (Y.W.) \\ * Correspondence: nikx@physics.ucsd.edu
}

Citation: Ni, K.; Qi, J.; Shockley, E.; Wei, Y. Sensitivity of a Liquid Xenon Detector to Neutrino-Nucleus Coherent Scattering and Neutrino Magnetic Moment from Reactor Neutrinos. Universe 2021, 7, 54. https://doi.org/10.3390/universe 7030054

Academic Editor: Clementina Agodi

Received: 29 December 2020

Accepted: 24 February 2021

Published: 3 March 2021

Publisher's Note: MDPI stays neutral with regard to jurisdictional claims in published maps and institutional affiliations.

Copyright: (c) 2021 by the authors. Licensee MDPI, Basel, Switzerland. This article is an open access article distributed under the terms and conditions of the Creative Commons Attribution (CC BY) license (https:/ / creativecommons.org/licenses/by/ $4.0 /)$.

\begin{abstract}
Liquid xenon is one of the leading targets to search for dark matter via its elastic scattering on nuclei or electrons. Due to their low-threshold and low-background capabilities, liquid xenon detectors can also detect coherent elastic neutrino-nucleus scattering (CEvNS) or neutrino-electron scattering. In this paper, we investigate the feasibility of a compact and movable liquid xenon detector with an active target mass of $O(10 \sim 100) \mathrm{kg}$ and single-electron sensitivity to detect CEvNS from anti-neutrinos from a nuclear reactor. Assuming a single- and few-electron background rate at the level achieved by the XENON10/100 experiments, we expect a 5- $\sigma$ detection of CEvNS with less than $400 \mathrm{~kg}$-days of exposure. We further investigate the sensitivity of such a detector to neutrino magnetic moment with neutrino electron scattering. If an electronic recoil background rate of $0.01 \sim 0.1$ events $/ \mathrm{keV} / \mathrm{kg} /$ day above $1 \mathrm{keV}$ can be achieved with adequate shielding, a liquid xenon detector can reach a neutrino magnetic moment sensitivity of $10^{-11} \mu_{B}$, which would improve upon the current most-constraining laboratory limits from the GEMMA and Borexino experiments. Additionally, such a detector would be able to probe the region compatible with a magnetic moment interpretation of the low-energy excess electronic recoil events recently reported by XENON1T.
\end{abstract}

Keywords: neutrino-nucleus interaction; solar neutrino; reactor neutrino; dark matter searches

\section{Introduction}

Detecting reactor neutrinos via neutrino-nucleus coherent elastic scattering (CEvNS) has been proposed in recent years using cryogenic bolometers [1,2], noble liquids [3,4], skipper CCDs [5], and low-threshold high-purity germanium detectors [6,7], thanks to the development of these sub-keV threshold nuclear recoil (NR) detectors for dark matter direct detection experiments. CEvNS is a Standard Model (SM) neutrino interaction process mediated by neutral weak currents [8], with a cross section approximately proportional to $N^{2}$, the square of the number of neutrons in the nucleus. The much larger cross section of CEvNS compared to other processes, such as the inverse beta decay (IBD), allows the detection of neutrinos with much smaller detectors. CEvNS was first observed by the COHERENT experiments $[9,10]$ using neutrinos from the Spallation Neutron Source. Detecting reactor neutrinos via the CEvNS process will not only utilize another neutrino source for the investigation of neutrino properties, but also provide potential applications in the safeguard of reactor fuels.

Noble liquid (liquid xenon and liquid argon) detectors have been successfully used in the dark matter direct detection experiments [11-14], with detector target masses ranging from $10 \mathrm{~kg}$ to 2 tonnes and nuclear recoil thresholds down to sub-keV with the ionizationonly channel capable to search for sub-GeV light dark matter. The technology has not been used for the CEvNS detection of reactor neutrinos. A two-phase liquid argon detector [15] was proposed in the last decade but the difficulty to achieve a lower background due to the radioactive Ar-39 at that time prevented further development. With the discovery of 
underground argon [16] with Ar-39 depleted, and the demonstration of sub-keV threshold of a liquid argon detector [13,17], the two-phase argon emission detector has regained interest towards reactor neutrino CEvNS detection, see, e.g., in [4].

For liquid xenon, although the XENON1T experiment [14] has achieved sub-keV threshold and a very low background, such a massive detector is not practical to be deployed near a reactor core due to its size, cost, complicated supporting systems, and the high muon-induced background at surface. An O(100) kg detector, the RED-100 [18], is being developed for the $\mathrm{CEvNS}$ detection of reactor neutrinos at the Kalinin nuclear power plant. With the high cross section of $\mathrm{CE} v \mathrm{NS}$, an even smaller liquid xenon target at $O(10)$ $\mathrm{kg}$ with better background control is possible for a first detection of reactor neutrinos via $\mathrm{CE} v \mathrm{NS}$. In addition to $\mathrm{CE} v \mathrm{NS}$, these compact liquid xenon detectors may also detect the neutrinos via electron-scattering and probe new physics related to neutrino properties, such as the neutrino magnetic moment.

In this paper, we study the feasibility of a 10-100 kg scale liquid xenon detector for $\mathrm{CE} v$ NS detection of reactor neutrinos with the realistic signal model for nuclear recoils in liquid xenon down to sub-keV and the most recent understanding of the relevant background down to single ionization electrons. We further estimate the sensitivity to neutrino magnetic moment (NMM) using such a compact and low-threshold detector near a nuclear reactor core. Detecting and measurement of the $\mathrm{CE} v \mathrm{NS}$ cross section and constraints on NMM from reactor neutrinos in liquid xenon will provide further input to the large liquid xenon experiments [19-21] for dark matter search, where the CEvNS from solar, atmospheric, and other neutrinos will be the ultimate background.

\section{Liquid Xenon for the Detection of CEvNS Events from Reactor Neutrinos}

For neutrinos scattering in a medium, the CEvNS event rate per unit mass and time is given by

$$
\frac{d R}{d E_{R}}=N_{T} \int_{E_{v}^{\min }}^{E_{v}^{\max }} \frac{d \Phi}{d E_{v}} \frac{d \sigma}{d E_{R}} d E_{v}
$$

where $N_{T}$ is the number of targets (electrons for ER and nuclei for NR) per unit mass in the medium, $E_{R}$ is the energy of the recoiling nucleus, and $E_{v}$ is the energy of the neutrino. The CEvNS cross section for a nucleus with $N, Z$ is given by [22]

$$
\frac{d \sigma}{d E_{R}}=\frac{G_{F}^{2}}{4 \pi}\left(N-Z\left(1-4 \sin ^{2} \theta_{w}\right)\right)^{2} m_{N}\left(1-\frac{m_{N} E_{R}}{2 E_{v}^{2}}\right) F^{2}\left(E_{R}\right)
$$

where $F^{2}\left(E_{R}\right)$ is the Helm form factor given by [23].

We use the reactor antineutrino spectra in Figure 3 of [24] (above $2 \mathrm{MeV}$ ) and [25] (below $2 \mathrm{MeV}$ ) and assume a fuel mixture of ${ }^{235} \mathrm{U}(26 \%),{ }^{238} \mathrm{U}(7.6 \%),{ }^{239} \mathrm{Pu}(51 \%)$, and ${ }^{241} \mathrm{Pu}$ $(14.8 \%)$ to obtain the overall antineutrino spectrum via

$$
S\left(E_{v}\right)=\sum_{i^{\text {th }} \text { isotope }} f_{i} \frac{d N_{i}}{d E_{v}}
$$

where $f_{i}$ and $\frac{d N_{i}}{d E_{v}}$ are the proportion of fissions and the antineutrino spectrum from the $i^{\text {th }}$ isotope, respectively. The formula for $\frac{d N_{i}}{d E_{v}}$ is given by [24]

$$
\frac{d N_{i}}{d E_{v}}=\sum_{n} Y_{n}(Z, A) \sum_{n, j} b_{n, j} P_{\bar{v}}\left(E_{\bar{v}}, Z\right)
$$

where $Y_{n}(Z, A)$ is the cumulative fission yield for the $n$th $\beta$ decaying nucleus, $b_{n, j}$ is the branching ratio to the $j$ th daughter, and $P$ is its antineutrino spectrum.

We then calculate the CEvNS rates from the reactor anti-neutrinos for several different noble element targets, shown in Figure 1 (top), assuming an anti-neutrino flux of $6.3 \times 10^{12} \mathrm{~cm}^{-2} \mathrm{~s}^{-1}$, which is approximately the flux at a distance of $25 \mathrm{~m}$ from the core 
of a $3 \mathrm{GW}$ thermal power reactor [15]. The energy of the nuclear recoils from CEvNS on a xenon target is smaller than $1 \mathrm{keV}$, making the detection very challenging. If a liquid xenon detector reaches a NR threshold of $300 \mathrm{eV}, \sim 10$ events $/ \mathrm{kg} /$ day would be detected. Other noble elements can also be used. The total number of CEvNS events from neutrinos on the lighter element is smaller, but the energy transfer is larger. In order to detect about 10 events $/ \mathrm{kg} /$ day, detectors with $\mathrm{Ne}, \mathrm{Ar}, \mathrm{Kr}$, or Xe elements all require sub-keV NR threshold. A detector with He target will require a larger target mass to detect similar number of events per kg per day. The detector technologies for different noble elements, including the cryogenics, photosensors, and purification are different. In this paper, we focus on the study of the liquid xenon target for which the detector technology was well developed.
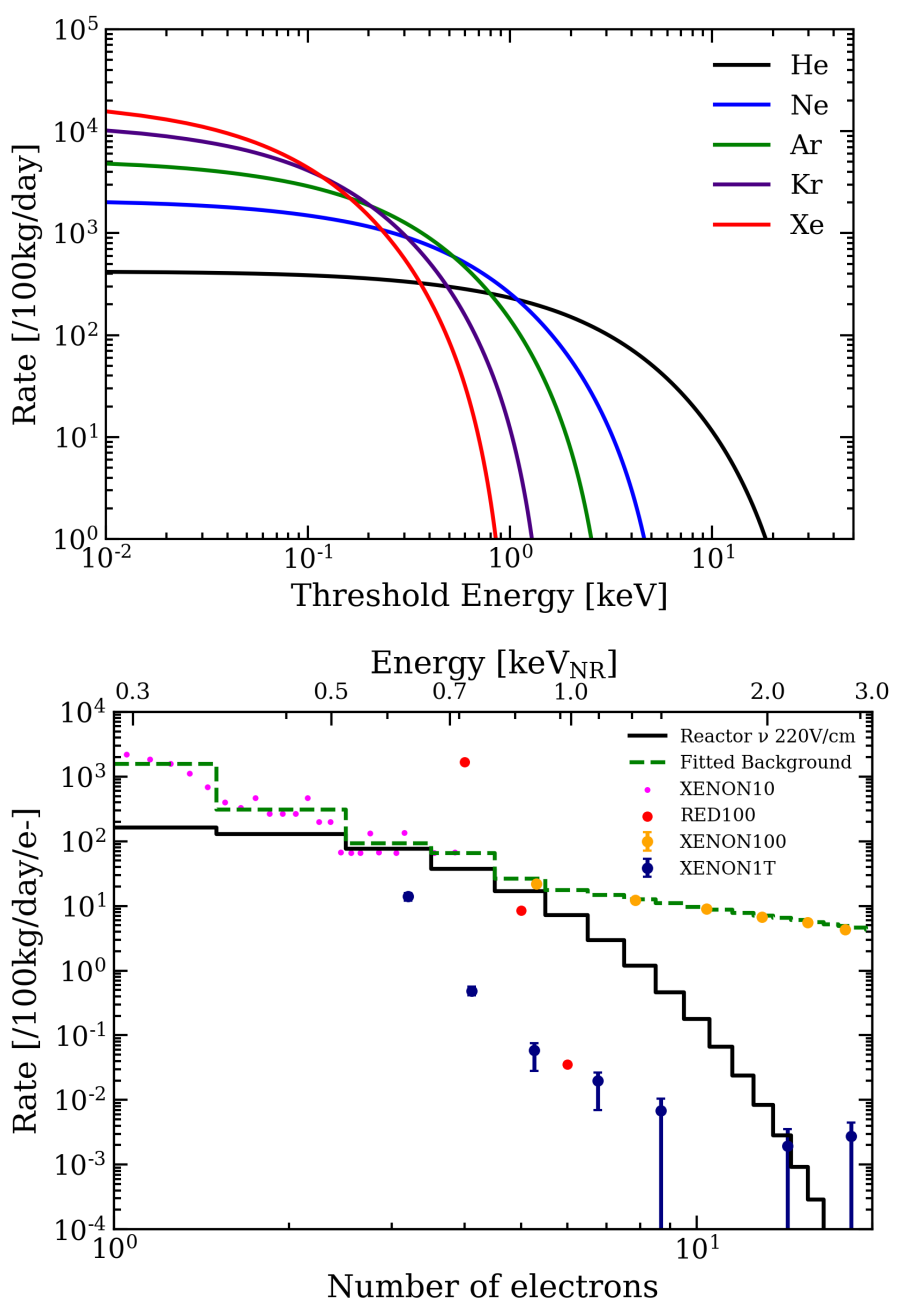

Figure 1. (Top): calculated $\mathrm{CE} v \mathrm{NS}$ rates in different noble elements from reactor anti-neutrinos with a flux of $6.3 \times 10^{12} \mathrm{~cm}^{-2} \mathrm{~s}^{-1}$ and assuming $7.6 \%{ }^{238} \mathrm{U}, 26 \%{ }^{235} \mathrm{U}, 14.8 \%{ }^{241} \mathrm{Pu}, 51 \%{ }^{239} \mathrm{Pu}$ fission elements composition. (Bottom): expected reactor anti-neutrino CE $v$ NS rates in number of detected electrons, compared with the background rates measured in several experiments. A "Fitted Background" (dashed green) from the XENON10/XENON100 is used as a benchmark background rate in a 10-100 kg liquid xenon detector for this study. The difference of background rates at this energy range from different experiments are due to their relative size and operation locations (see text for details).

The responses of liquid xenon to low-energy nuclear recoils, from the interaction of particles, such as neutrons, neutrinos or the hypothetical dark matter candidate Weakly Interacting Massive Particles (WIMPs), and electronic recoils, from gamma rays or electrons, were well investigated in the last two decades, especially using the liquid xenon time 
projection chambers (LXeTPCs), see, e.g., in [26,27]. LXeTPCs extract two signals for each nuclear or electronic recoil interacting in the medium. One is the prompt scintillation signal (S1), from the direct excitation and recombination of electron-ion pairs. The other is a delayed signal (S2) from ionization electrons escaping the recombination and amplified through electroluminescence in the gas phase. Both S1 and S2 signals are recorded by arrays of photosensors, usually photomultiplier tubes (PMTs). The energy threshold of LXeTPCs in standard analyses is set by the S1 light collection efficiency to 2-3 photoelectrons (PE). In contrast, thanks to the inherent $\mathrm{S} 2$ amplification, even a single extracted electron can produce $\sim 20-100 \mathrm{PE}$ in S2, which thus allows for a lower energy threshold when using this channel alone ("S2-only" or "ionization only" analysis). This technique can detect a "singleelectron", from the ionization of a $300 \mathrm{eV}$ nuclear recoil in liquid xenon, with full efficiency, as demonstrated by a latest measurement [28] employing this technique. Below $300 \mathrm{eV}$, the efficiency falls off quickly as such a low energy nuclear recoil would not produce even a single ionization electron.

We convert the energy spectrum to the ionization spectrum using the latest measured charge yield, $Q_{y}\left(E_{R}\right)$ which is the expected number of ionization electrons produced per unit kinetic energy released in liquid xenon by the nuclear recoils, from [28]. The expected number of electrons for a given energy is then $E_{R} Q_{y}\left(E_{R}\right)$, and is assumed to follow a Poisson distribution. Therefore, the rate of $\mathrm{CE} v \mathrm{NS}$ events per mass/time/electrons is

$$
R\left(n_{e}\right)=\int \frac{d R}{d E_{R}} \frac{e^{-E_{R} Q_{y}\left(E_{R}\right)}\left(E_{R} Q_{y}\left(E_{R}\right)\right)^{n_{e}}}{n_{e} !} d E_{R} .
$$

Figure 1 (bottom) provides our calculated rate as a function of number of detectable electrons produced by the $\mathrm{CE} v \mathrm{NS}$ process of reactor anti-neutrinos in the liquid xenon detector. We use the charge yield at $220 \mathrm{~V} / \mathrm{cm}$ [28] measured in liquid xenon and expect not much change at higher drift fields. According to the calculation, about $440 \mathrm{CE} v \mathrm{NS}$ events, among which about 270 events producing at least two ionization electrons, are expected in a $100 \mathrm{~kg}$-days exposure in a liquid xenon target. The highest rate is at the single and double ionization electron level. The high event rate from reactor neutrino coherent scattering in the liquid xenon detector makes it feasible to monitor the reactor anti-neutrino flux in real time. For example, a $100 \mathrm{~kg}$ mass detector can measure the daily event rate with $\sim 5 \%$ statistical uncertainty, assuming the background is negligible.

We compare the calculated rate with the single-and-few electron background rates measured in the XENON10 [11,29], XENON100 [12], and XENON1T [14] experiments. These are the three generations of dark matter search experiments using LXeTPC technology with target masses at about $15 \mathrm{~kg}, 60 \mathrm{~kg}$ and 2 tonnes, respectively, all located at Gran Sasso Underground Laboratory in Italy. We also compare the calculated rate with a special run of the RED-100 detector [18] to measure the "spontaneous" single electron rate at the surface. RED-100 is also a LXeTPC with about $160 \mathrm{~kg}$ active liquid xenon target, specially designed for the detection of reactor neutrinos at the Kalinin nuclear power plant in Russia. While the calculated rate for single and double electrons from $\mathrm{CE} v \mathrm{NS}$ is still lower than the current lowest single-and-double electron background rate achieved [29], the rate at 3-5 electrons is comparable to the achieved background in a 10-100 kg LXe detector. For comparison, the background rate caused by accidental coincidence of "spontaneous" single electrons measured in the RED-100 detector at surface [18] is lower than the calculated CEvNS rate at or above 5 electrons. The XENON1T [14] detector achieved much lower rate above 3 electrons, but the detector is too large to be placed near a reactor core.

Investigation of the sources of the single-electron background in liquid xenon detectors was carried out in the last few years [30,31], pointing to several origins: one is related to the impurities [32] in liquid xenon which could attach drifting electrons and later release them, another is from the electrons trapped at the liquid-gas interface [33] in the two-phase emission detector and later released to the gas phase, and other possible impurities that would release electrons spontaneously. For the impurity related single electron background, a significant reduction of impurity compared to the current state-of-art liquid xenon purity 
is required. In addition to a fast circulation and purification speed with gaseous or liquid xenon, attempts to contain the liquid xenon target in a sealed chamber to reduce the outgassing contaminant were tried [34,35]. For the electrons trapped below the liquid surface, a stronger extraction field [36] is needed to reduce them. However, there are limitations of supplying strong field in the liquid-gas interface. One alternative solution is to avoid the liquid-gas interface by amplifying the single-electron signals through electroluminescence in liquid xenon directly [37-39].

\section{Discovery Significance to Detect Reactor Neutrinos}

In this section, we estimate the discovery significance to detect reactor neutrinos by considering the expected $\mathrm{CE} v \mathrm{NS}$ events as calculated in Figure 1 (bottom). The single-andmultiple electron background in the liquid xenon detectors are difficult to be modeled. Instead, we use a fitted background from XENON10/XENON100 measured rates as a benchmark background assumption. The background for XENON10 is efficiency corrected and fitted with four Gaussians (representing 1, 2, 3, and $4 e^{-}$) and the XENON100 background is fitted with a power law. As the single-electron rate is much higher than the expected background, we performed our study using a threshold of 2, 3, 4, 5e $e^{-}$with an upper range of 10 electrons, above which the CEvNS signal rate is negligible. We use an uncertainty of $8 \%$ for the background fluctuation, according to the systematic uncertainty from XENON100 [12].

The likelihood function used is

$$
L(\mu, f)=\frac{e^{-(\mu s+f b)}(\mu s+f b)^{N_{o b s}}}{N_{o b s} !} \frac{1}{\sqrt{2 \pi} \sigma_{b}} e^{-(f-1)^{2} / 2 \sigma_{b}^{2}}
$$

where $\mu$ and $f$ represent the signal and background normalizations, respectively, and $\sigma_{b}$ is the background systematic uncertainty. The test statistic used here is the $q_{0}$ statistic which is

$$
q_{0}=-2 \ln \frac{L(0, \hat{f})}{L(\hat{\mu}, \hat{f})}
$$

where the hats denote the values of $\mu$ and $f$ that maximize the likelihood function, and $\hat{f}$ is the value of $f$ that maximizes the function when $\mu=0$. The median $p$-value and significance are given by

$$
p=\int_{\operatorname{med}\left(q_{0, s+b}\right)}^{\infty} f\left(q_{0} \mid \mu=0\right) d q_{0}, \quad \text { Significance }=\Phi^{-1}(1-p)
$$

Here, $\operatorname{med}\left(q_{0, s+b}\right)$ is the median $q_{0}$ when signals are injected, and $f\left(q_{0} \mid \mu=0\right)$ is the background only (no signal injected) $q_{0}$ distribution.

The expected significance of $C E v$ NS detection is shown in Figure 2 for four different thresholds at 2,3,4, and 5 electrons. The detection sensitivity is more significant using events with 2 to 5 electrons due to the high signal rate in this signal region. If the background level at 2 to 5 electrons cannot be achieved in a surface operation compared to the underground operation of XENON10/100, using the 5-10 electrons region of interest (RoI) would also give a 5- $\sigma$ detection within $400 \mathrm{~kg}$-days of exposure. Thus a detector with 10 to $100 \mathrm{~kg}$ target mass is expected to achieve this significance very quickly. Further reduction of the background below 5 electrons will make it attractive to detect reactor CEvNS events with a compact $O(10)-\mathrm{kg}$ detector. A liquid xenon detector with 2-3 electron threshold and similar background as XENON10 will be able to achieve a 5- $\sigma$ detection within $100 \mathrm{~kg}$-days of exposure. 


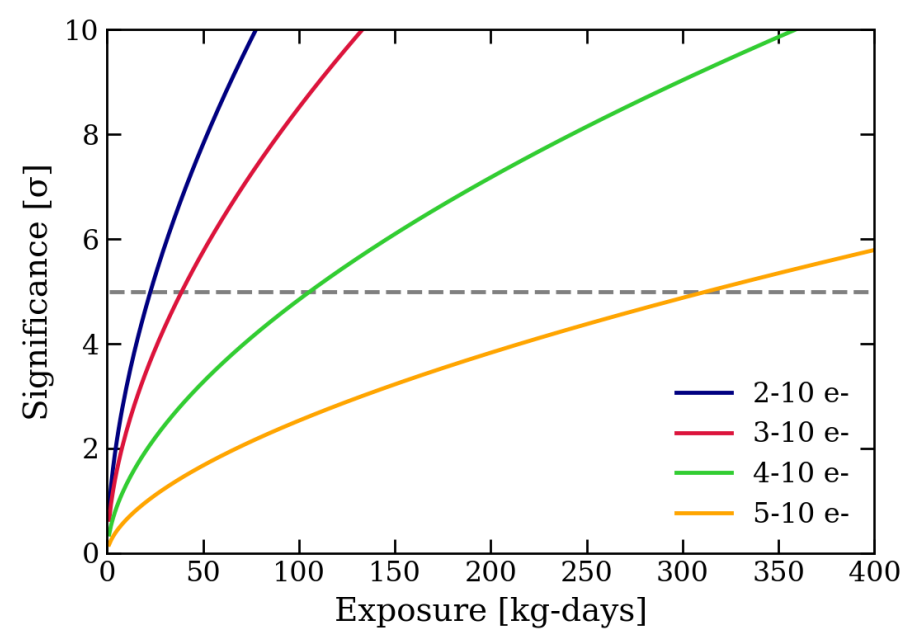

Figure 2. Significance to detect neutrino $\mathrm{CE} v \mathrm{NS}$ as a function of exposure using a liquid xenon detector placed about $25 \mathrm{~m}$ away near the core of a $3 \mathrm{GW}$ thermal power reactor, assuming different achievable detector thresholds and a single-and-few electrons background comparable to that measured in XENON10/100.

\section{Sensitivity to Neutrino Magnetic Moment}

With a precise measurement of CEvNS in xenon and liquid xenon's capability to suppress electronic recoil background at keV-scale energies, we can also probe new physics beyond the Standard Model [40], including a search for a large neutrino magnetic moment [41], neutrino electric millicharge [42], neutrino non-standard interactions [43], and sterile neutrinos [44]. A large neutrino magnetic moment of neutrinos from the Sun could explain the recently observed electronic recoil excess events in the XENON1T experiment [45] using a liquid xenon target. In this paper, we study the sensitivity of such a liquid xenon detector to the neutrino magnetic moment using reactor neutrinos.

The non-zero mass of neutrinos imply a non-zero neutrino magnetic moment $\mu_{v}$, although the value is very small $\sim 3 \times 10^{-19} \mu_{B}\left[m_{v} / 1 \mathrm{eV}\right]$ [46], where $\mu_{B}$ is the Bohr magneton, in the minimally extended Standard Model with non-zero neutrino mass. A "naturalness" upper bound on $\mu_{v}$ for Dirac neutrinos, generated by physics above the scale of electroweak symmetry breaking, is $\mu_{v}<10^{-15} \mu_{B}$ [47,48]. For Majorana neutrinos, on the other hand, the theoretical bound for $\mu_{v}$ is much weaker [48], comparable to the present experimental limits from the Borexino solar neutrino experiment [49]. Recently, the XENON1T experiment observed a low-energy excess of electronic recoil events that can be interpreted as from solar neutrinos with a large magnetic moment between $(1.4-2.9) \times 10^{-11} \mu_{B}$ $(3.2 \sigma)$ [45]. Although the value is in strong tension with the indirect astrophysical bounds of $\sim 10^{-12} \mu_{B}[50,51]$ and globular clusters, a reactor neutrino experiment can check the hypothetical large neutrino magnetic moment independently. Note that, while ignored here for simplicity, neutrino mixing does have an impact on the NMM interaction, implying that constraints on this effective, scalar $\mu_{v}$ are not directly comparable for reactor and solar neutrino sources. For more details, see in [52].

So far, the most stringent NMM upper limit from reactor neutrinos is $2.9 \times 10^{-11} \mu_{B}$ from the GEMMA experiment [53], which used a $1.5 \mathrm{~kg}$ high-purity germanium (HPGe) detector placed at a distance of $14 \mathrm{~m}$ from the $3 \mathrm{GW}$ th reactor core, exposing to an antineutrino flux of $2.7 \times 10^{13} \mathrm{~cm}^{-2} \mathrm{~s}^{-1}$. Here, we first consider the sensitivity of a $10 \mathrm{~kg}$ scale liquid xenon detector to NMM from neutrino-electron elastic scattering from reactor neutrinos with the same flux $\left(6.3 \times 10^{12} \mathrm{~cm}^{-2} \mathrm{~s}^{-1}\right)$ assumed in Section 2 using total energy above $1 \mathrm{keV}$, where electronic recoil background is well understood, then compare the sensitivity to NMM using the low energy ionization-only (S2-only) approach, and different signal and background assumptions. 
The event rate of neutrino-electron elastic scattering can be calculated through the weak interaction cross section, given by [54]

$$
\frac{d \sigma_{W}}{d E_{R}}=\frac{G_{F}^{2} m_{e}}{2 \pi}\left[4 s^{4}+\left(1+2 s^{2}\right)^{2}\left(1-\frac{E_{R}}{E_{v}}\right)^{2}-2 s^{2}\left(1+2 s^{2}\right) \frac{m_{e} E_{R}}{E_{v}^{2}}\right]
$$

and the electromagnetic (EM) interaction with a magnetic moment $\mu_{v}$ [53],

$$
\frac{d \sigma_{E M}}{d E_{R}}=\pi r_{0}^{2}\left(\frac{\mu_{v}}{\mu_{B}}\right)^{2}\left(\frac{1}{E_{R}}-\frac{1}{E_{v}}\right)
$$

where $E_{R}$ is the electronic recoil energy, the Standard Model prediction of the weak mixing angle $s^{2}=\sin ^{2} \theta_{W}=0.23152 \pm 0.00005$ [55], and $r_{0}=2.818 \times 10^{-13} \mathrm{~cm}$ is the classical electron radius.

For both the Standard Model $v$-e scattering and the EM neutrino magnetic moment interaction, we account for the fact that the electrons in xenon are bounded in atomic shells. That is, events with recoil energies below a given electron binding energy cannot ionize that electron [56], which effectively reduces the scattering cross section. We therefore split the energy spectra by the energies of each shell and scale each segment down by the number of electrons available divided by $Z$. This accounts for the saw-tooth shape in Figure 3 (bottom), see in [56] for a more detailed calculation.
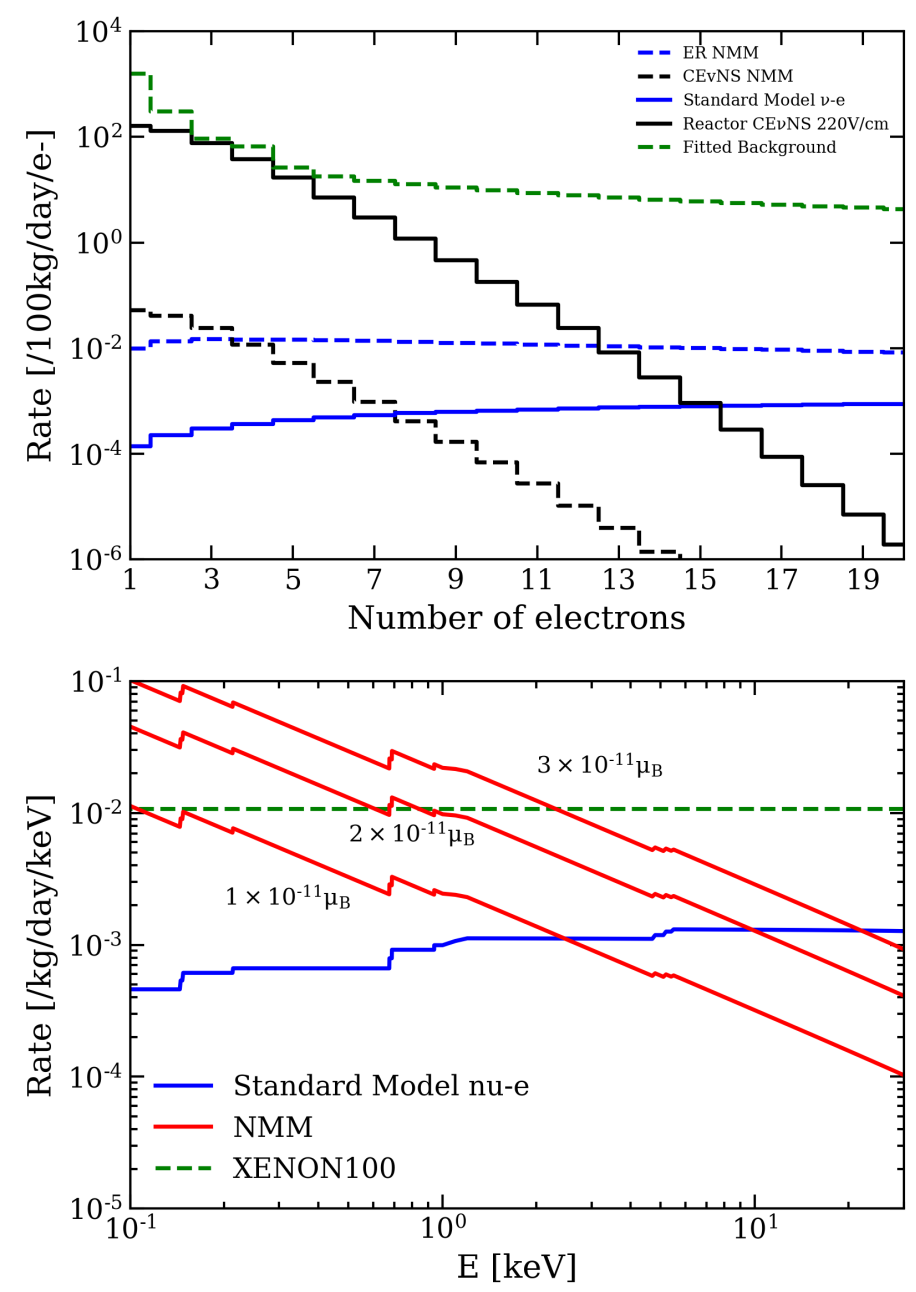

Figure 3. (Top): the SM (solid lines) and NMM contributions (black and blue dashed lines, assuming $\mu_{v}=10^{-11} \mu_{B}$ ) to $\mathrm{CE} v$ NS (black) and neutrino-electron scattering (green) channels for low-energy. events with "ionization (S2) only". The "fitted background" (dashed green) from measured rates in 
XENON10/100 is shown as a benchmark background rate for sensitivity study. (Bottom): expected SM (blue) and NMM (red) contributions to the neutrino-electron scattering event rates in "Total Energy" channel. At above $1 \mathrm{keV}$, the electron recoils typically generate both $\mathrm{S} 1$ and S2 signals and the "Total Energy" background can be much better controlled. A benchmark electronic recoil background rate (dashed green) of $10 \mathrm{mDRU}\left(1 \mathrm{mDRU}=10^{-3}\right.$ events $\left./ \mathrm{keV} /\right)$ from XENON100 [57] is plotted for comparison.

The contribution of a large $\mu_{v}$ to the neutrino electron scattering rate increases rapidly towards lower energy, even below $1 \mathrm{keV}$. However, a two-phase xenon TPC's detection efficiency with the primary scintillation light (S1) drops quickly for events below $1 \mathrm{keV}$, as shown in, e.g., [45]. An ionization-only (S2-only) [14] analysis improves the detection efficiency for low-energy events close to $100 \%$ due to its capability to detect a single electron, which has an equivalent energy to $O(10) \mathrm{eV}$ of electron recoil or $O(100) \mathrm{eV}$ of nuclear recoil events. On the other hand, losing the S1 signals limits the capability to locate the event $Z$ positions resulting in worse background suppression.

The $90 \%$ upper limit to $\mu_{v}$ is thus calculated in two ways. The first way is to use the energy spectrum from $1 \mathrm{keV}$ to $10 \mathrm{keV}$ (Figure 3 bottom) where the electronic recoil background is well understood and can be controlled, the second way is to use the signal and backgrounds from the ionization-only channel from 10-20 ionization electrons as shown in Figure 3 (top). For the ionization-only approach, the ER backgrounds are converted into the number of ionization electrons via the NEST charge yields [58]. The background rate increases substantially due to the reduced capability of rejecting background events based on their position distribution in the sensitive target. In addition to the instrumental background at single to a few electron level, the Standard Model CEvNS also contributes significantly to the ionization-only event rate below $10 \mathrm{e}^{-}$. A large neutrino magnetic moment would also give a small enhancement of the CE $v$ NS event rate according to [59]

$$
\frac{d \sigma_{v-N}^{\text {mag. }}}{d E_{R}}=\frac{\pi \alpha^{2} \mu_{v}^{2} Z^{2}}{m_{e}^{2}}\left(\frac{1}{E_{R}}-\frac{1}{E_{v}}+\frac{E_{R}}{4 E_{v}^{2}}\right) F^{2}\left(E_{R}\right)
$$

The binned likelihood function used to calculate the $90 \%$ upper limit of $\mu_{v}$ is

$$
-2 \ln \mathcal{L}(\mu, \vec{f})=2\left(\mu s^{t o t}+\vec{f} \cdot \vec{b}^{t o t}\right)-2 \sum_{i=1}^{N_{b i n s}} N_{o b s, i} \ln \left[\mu s_{i}+\vec{f} \cdot \vec{b}_{i}\right]+\sum_{j=1}^{N_{b g}} \frac{\left(f_{j}-1\right)^{2}}{\sigma_{j}^{2}}
$$

where $\vec{f}$ represents the background rate multipliers, $\vec{b}$ represents the background components, $\mu$ is the signal multiplier, and $\sigma$ represents the background systematic uncertainties (assumed to be $10 \%$ for this study). The label $i$ runs over each bin; this calculation bins the spectra per number of electrons produced from 10-20 $\mathrm{e}^{-}$when using the S2 only channel and $1 \mathrm{keV}$ wide bins from 1 to $10 \mathrm{keV}$ when using the total energy spectrum. The test statistic used is $\tilde{q}_{\mu}$ as described in [60].

To predict the median $90 \%$ upper limit on the neutrino magnetic moment, we calculate the distribution of $\tilde{q}_{\mu}$ with no signals injected for a given $\mu$ by simulating 10,000 toy experiments to find $\operatorname{med}\left(\tilde{q}_{\mu, o b s}\right)$. The $\tilde{q}_{\mu}$ distribution for experiments with a signal injected of strength $\mu$ is assumed to be a half-chi-squared distribution with one degree of freedom, and the integral of this distribution with signals injected from $\operatorname{med}\left(\tilde{q}_{\mu, o b s}\right)$ to infinity gives the $p$-value of interest [60]. Then, the value of $\mu$ which gives a $p$-value of $10 \%$ is the $90 \%$ upper limit of $\mu_{v}^{2}$. Note here that [60] has a slightly different asymptotic formula for $\tilde{q_{\mu}}$ with an injected signal, but the difference is negligible.

From the calculation, as shown in Figure 4, we find that the total energy $(1-10 \mathrm{keV})$ channel is very sensitive to the NMM if a low background rate similar to XENON100 (10 mDRU, $1 \mathrm{mDRU}=10^{-3}$ events $/ \mathrm{keV} / \mathrm{kg} /$ day) can be achieved. A $90 \%$ upper limit about $10^{-11} \mu_{B}$ can be achieved with about $3500 \mathrm{~kg}$-days of exposure near the reactor. A ten 
times higher background (100 mDRU) would still make it possible to achieve a limit better than GEMMA, the current most-constraining reactor experiment for $\mu_{v}$.

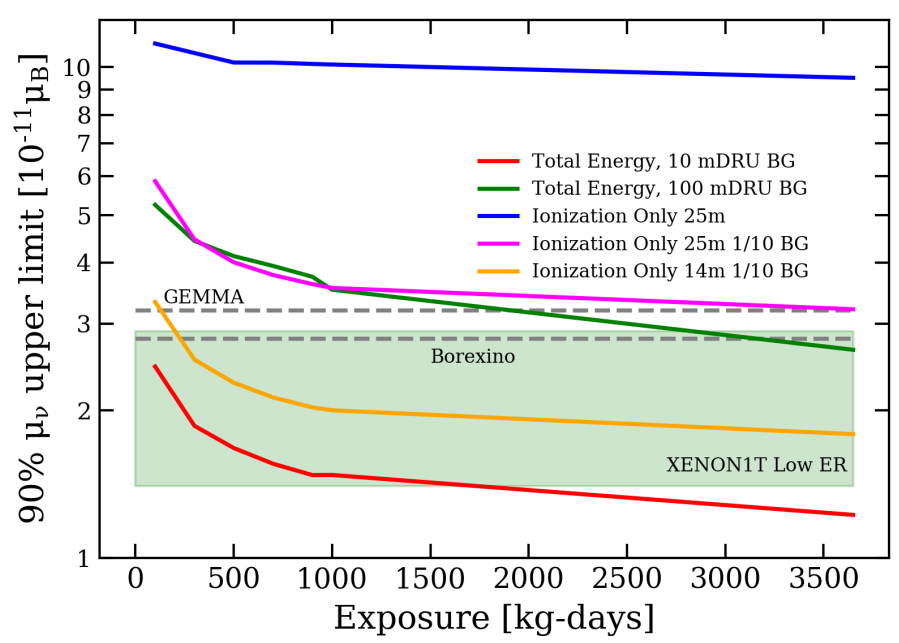

Figure 4. Sensitivity (median $90 \%$ upper limit) to neutrino magnetic moment from reactor neutrinos interaction in a liquid xenon detector, using either the ionization-only analysis for sub-keV energy events or the total energy for events above $1 \mathrm{keV}$. For the ionization-only approach, we compare the sensitivity between the standard assumptions (see Section 2) and more aggressive assumptions with reduced $(1 / 10)$ S2-only background or placing the detector closer to the reactor core, from $25 \mathrm{~m}$ to $14 \mathrm{~m}$, to increase the neutrino flux. For the "total energy" analysis, we assume $100 \mathrm{mDRU}$ (10 mDRU) ER background, which is achievable in a liquid xenon detector with 10 100 kg target mass. The upper limits (dashed horizontal lines) from GEMMA [53] and Borexino [49] experiments, as well as the allowed range (shaded green) from the excess of low energy electronic recoil events in XENON1T [45] are plotted for comparison.

The sensitivity using the ionization-only channel is limited by the high background with only S2 signals. We also calculated the ionization-only sensitivity in the case that the background can be reduced by a factor of ten with the detector developments mentioned in Section 2 to reduce the single-and-few electron background. This is assuming that the impurity in liquid xenon can be reduced by a factor of ten or the electrons trapped at the liquid-gas interface can be reduced by a factor of ten. Due to the limitation of space near the reactor core, we keep a conservative distance of $25 \mathrm{~m}$ from the reactor core for the standard rate calculation. However, if space closer to the reactor core, e.g., $14 \mathrm{~m}$, is available, the flux can be increased thus increasing the sensitivity to NMM. In any case, we get a more stringent limit by using the total energy, which necessitates the detection of the primary (S1) scintillation signal. The "total energy" channel requires more coverage of photosensors thus increasing the cost and radioactivity background from photosensor-related materials.

\section{Summary}

In this work, we have described the expected sensitivity of an O(10-100)-kg LXeTPC to both CEvNS and an anomalous neutrino magnetic moment using reactor neutrinos. If the backgrounds can be sufficiently reduced at the $<5$-electron level, a $10 \mathrm{~kg}$ LXeTPC is expected to observe $\mathrm{CE} v \mathrm{NS}$ at a $O(\mathrm{GW})$ reactor with high significance. In the conservative scenario where these low-energy backgrounds are not suppressed relative to existing experiments, a significant detection is still expected with a $O(100)-\mathrm{kg}$ detector. Such an experiment might be the first to detect $\mathrm{CE} v \mathrm{NS}$ with liquid xenon, which would be an important measurement for constraining the Standard Model prediction of the CE $v \mathrm{NS}$ cross sections.

We also present the sensitivity of such a detector to an anomalous neutrino magnetic moment, which if observed would point to physics beyond the Standard Model. For this 
signal we show that, on account of the improved background suppression, the sensitivity is best if both scintillation and ionization signals are considered, despite the higher energy threshold. With a $3500 \mathrm{~kg}$-days exposure near the reactor, we expect a LXeTPC to probe $\mu_{v} \sim 10^{-11} \mu_{B}$. This would be a factor of $\sim 2$ improvement relative to the GEMMA result, which is currently the strongest limit using reactor neutrinos. Such a xenon experiment would also cover the parameter space consistent with a magnetic moment interpretation of the recent XENON1T excess [45], and so could either rule out or confirm this interpretation using a different source of neutrinos.

Author Contributions: Conceptualization, K.N. and Y.W.; software and analysis, J.Q.; validation, E.S.; writing—original draft preparation, K.N. and J.Q.; writing—review and editing, Y.W. and E.S.; supervision, project administration, and funding acquisition, K.N. All authors have read and agreed to the published version of the manuscript.

Funding: This research is sponsored by the US Defense Advanced Research Projects Agency under grant number HR00112010009, the content of the information does not necessarily reflect the position or the policy of the Government, and no official endorsement should be inferred.

Acknowledgments: The authors would like to thank Cheng-Pang Liu and Jiunn-Wei Chen for discussions regarding the neutrino rate calculations.

Conflicts of Interest: The authors declare no conflict of interest.

\section{References}

1. Billard, J.; Carr, R.; Dawson, J.; Figueroa-Feliciano, E.; Formaggio, J.A.; Gascon, J.; Heine, S.T.; De Jesus, M.; Johnston, J.; Lasserre, T.; et al. Coherent Neutrino Scattering with Low Temperature Bolometers at Chooz Reactor Complex. J. Phys. G 2017, 44, 105101. [CrossRef]

2. Agnolet, G.; Baker, W.; Barker, D.; Beck, R.; Carroll, T.J.; Cesar, J.; Cushman, P.; Dent, J.B.; Rijck, S.D.; Dutta, B.; et al. Background Studies for the MINER Coherent Neutrino Scattering Reactor Experiment. Nucl. Instrum. Meth. A 2017, 853, 53-60. [CrossRef]

3. Akimov, D.Y.; Berdnikova, A.B.; Belov, V.A.; Bolozdynya, A.I.; Burenkov, A.A.; Efremenko, Y.V.; Gusakov, Y.V.; Etenko, A.V.; Kaplin, V.A.; Khromov, A.V.; et al. RED-100 detector for the first observation of the elastic coherent neutrino scattering off xenon nuclei. J. Phys. Conf. Ser. 2016, 675, 012016. [CrossRef]

4. Wei, Y.T.; Guan, M.Y.; Liu, J.C.; Yu, Z.Y.; Yang, C.G.; Guo, C.; Xiong, W.X.; Gan, Y.Y.; Zhao, Q.; Li, J.J. Prospects of detecting the reactor $\overline{v_{e}}$-Ar coherent elastic scattering with a low threshold dual-phase argon time projection chamber at Taishan. arXiv 2020, arXiv:2012.00966.

5. Aguilar-Arevalo, A.; Bertou, X.; Bonifazi, C.; Cancelo, G.; Castañeda, A.; Vergara1, B.C.; Chavez, C.; D'Olivo1, J.C.; Anjos, J.C.; Estrada, J.; et al. Exploring low-energy neutrino physics with the Coherent Neutrino Nucleus Interaction Experiment. Phys. Rev. D 2019, 100, 092005. [CrossRef]

6. Buck, C.; Fülber, K.; Hakenmüller, J.; Heusser, G.; Lindner, M.; Maneschg, W.; Rink, T.; Strecker, H.; Schierhuber, T.; Wagner, V.; et al. A novel experiment for coherent elastic neutrino nucleus scattering: CONUS. J. Phys. Conf. Ser. 2020, 1342, 012094. [CrossRef]

7. Strauss, R.; Rothe, J.; Petricca, F.; Schönert, S. The v-cleus experiment: Gram-scale cryogenic calorimeters for a discovery of coherent neutrino scattering. J. Phys. Conf. Ser. 2020, 1342, 012132. [CrossRef]

8. Freedman, D.Z. Coherent effects of a weak neutral current. Phys. Rev. D 1974, 9, 1389-1392. [CrossRef]

9. Akimov, D.; Albert, J.B.; An, P.; Awe, C.; Barbeau, P.S.; Becker, B.; Belov, V.; Brown, A.; Bolozdynya, A.; Cabrera-Palmer, B.; et al. Observation of Coherent Elastic Neutrino-Nucleus Scattering. Science 2017, 357, 1123-1126. [CrossRef]

10. Akimov, D.; Albert, J.B.; An, P.; Awe, C.; Barbeau, P.S.; Becker, B.; Belov, V.; Blackston, M.A.; Blockland, A.; Bolozdynya, A.; et al. First Detection of Coherent Elastic Neutrino-Nucleus Scattering on Argon. arXiv 2020, arXiv:2003.10630.

11. Angle, J.; Aprile, J.; Arneodo, F.; Baudis, L.; Bernstein, A.; Bolozdynya, A.I.; Coelho, L.C.C.; Dahl, C.E.; DeViveiros, L.; FerellaWagner, A.D.; et al. Search for light dark matter in XENON10 data. Phys. Rev. Lett. 2011, 107, 051301. [CrossRef] [PubMed]

12. Aprile, E.; Aalbers, J.; Agostini, F.; Alfonsi, M.; Amaro, F.D.; Anthony1, M.; Arneodo, F.; Barrow, P.; Baudis, L.; Bauermeister, B.; et al. Low-mass dark matter search using ionization signals in XENON100. Phys. Rev. 2016, D94, 092001. [CrossRef]

13. Agnes, P.; Albuquerque, I.F.M.; Alexander, T.; Alton, A.K.; Araujo, G.R.; Asner, D.M.; Ave, M.; Back, H.O.; Baldin, B.; Batignani, G.; et al. Low-Mass Dark Matter Search with the DarkSide-50 Experiment. Phys. Rev. Lett. 2018, 121, 081307. [CrossRef] [PubMed]

14. Aprile, E.; Aalbers, J.; Agostini, F.; Alfonsi, M.; Althueser, L.; Amaro, F.D.; Antochi, V.C.; Angelino, E.; Arneodo, F.; Barge, D.; et al. Light Dark Matter Search with Ionization Signals in XENON1T. Phys. Rev. Lett. 2019, 123, 251801. [CrossRef] [PubMed]

15. Hagmann, C.; Bernstein, A. Two-phase emission detector for measuring coherent neutrino-nucleus scattering. IEEE Trans. Nucl. Sci. 2004, 51, 2151-2155. [CrossRef]

16. Back, H.O.; Calaprice, F.; Condon, C.; de Haas, E.; Ford, R.; Galbiati, C.; Goretti, A.; Hohman, T.; Inanni, A.; Loer, B.; et al. First Large Scale Production of Low Radioactivity Argon From Underground Sources. arXiv 2012, arXiv:1204.6024. 
17. Sangiorgio, S.; Joshi, T.; Bernstein, A.; Coleman, J.; Foxe, M.; Hagmann, C.; Jovanovic, I.; Kazkaz, K.; Mavrokoridis, K.; Mozin, V.; et al. First demonstration of a sub-keV electron recoil energy threshold in a liquid argon ionization chamber. NIM A 2013, 728, 69-72. [CrossRef]

18. Akimov, D.Y.; Belov, V.A.; Bolozdynya, A.I.; Dolgolenko, A.G.; Efremenko, Y.V.; Etenko, A.V.; Galavanov, A.V.; Gouss, D.V.; Gusakov, Y.V.; Kdib, D.E.; et al. First ground-level laboratory test of the two-phase xenon emission detector RED-100. arXiv 2019, arXiv:1910.06190.

19. Akerib, D.S.; Akerlof, C.W.; Alsum, S.K.; Araújo, H.M.; Arthurs, M.; Bai, X.; Bailey, A.J.; Balajthy, J.; Balashov, S.; Bauer, D.; et al. Projected WIMP Sensitivity of the LUX-ZEPLIN (LZ) Dark Matter Experiment. arXiv 2018, arXiv:1802.06039.

20. Aprile, E.; Aalbers, J.; Agostini, F.; Alfonsi, M.; Althueser, L.; Amaro, F.D.; Antochi, V.C.; Angelino, E.; Angevaare, J.R.; Arneodo, F.; et al. Projected WIMP sensitivity of the XENONnT dark matter experiment. J. Cosmol. Astropart. Phys. 2020, 11, 31. [CrossRef]

21. Aalbers, J.; Agostini, F.; Alfonsi, M.; Amaror, F.D.; Amsler, C.; Aprile, E.; Arazi, L.; Arneodo, F.; Barrow, P.; Baudis, L.; et al. DARWIN: Towards the ultimate dark matter detector. J. Cosmol. Astropart. Phys. 2016, 11, 17. [CrossRef]

22. Essig, R.; Sholapurkar, M.; Yu, T.T. Solar neutrinos as a signal and background in direct-detection experiments searching for sub-GeV dark matter with electron recoils. Phys. Rev. D 2018, 97, 095029. [CrossRef]

23. Lewin, J.; Smith, P. Review of mathematics, numerical factors, and corrections for dark matter experiments based on elastic nuclear recoil. Astropart. Phys. 1996, 6, 87-112. [CrossRef]

24. Hayes, A.C.; Vogel, P. Reactor Neutrino Spectra. Ann. Rev. Nucl. Part. Sci. 2016, 66, 219-244. [CrossRef]

25. Ishimoto, S.; Omori, T.; Arima, H.; Ishibashi, K. Simple Calculation of Reactor Antineutrino Energy Spectrum by the Use of Nuclear Data Libraries. J. Nucl. Sci. Technol. 2002, 39, 670-672. [CrossRef]

26. Aprile, E.; Angle, J.; Arneodo, F.; Baudis, L.; Bernstein, A.; Bolozdynya, A.; Brusov, P.; Coelho, L.C.C.; Dahl, C.E.; DeViveiros, L.; et al. Design and Performance of the XENON10 Dark Matter Experiment. Astropart. Phys. 2011, 34, 679-698. [CrossRef]

27. Aprile, E.; Arisaka, K.; Arneodo, F.; Askin, A.; Baudis, L.; Behrens, A.; Brown, E.; Cardoso, J.M.R.; Choi, B.; Clin, D.; et al. The XENON100 Dark Matter Experiment. Astropart. Phys. 2012, 35, 573-590. [CrossRef]

28. Lenardo, B.; Xu, J.; Pereverzev, S.; Akindele, O.A.; Naim, D.; Kingston, J.; Bernstein, A.; Kazkaz, K.; Tripathi, M.; Awe, C.; et al. Measurement of the ionization yield from nuclear recoils in liquid xenon between $0.3-6 \mathrm{keV}$ with single-ionization-electron sensitivity. arXiv 2019, arXiv:1908.00518.

29. Essig, R.; Manalaysay, A.; Mardon, J.; Sorensen, P.; Volansky, T. First Direct Detection Limits on sub-GeV Dark Matter from XENON10. Phys. Rev. Lett. 2012, 109, 021301. [CrossRef] [PubMed]

30. Aprile, E.; Alfonsi, M.; Arisaka, K.; Arneodo, F.; Balan, C.; Baudis, L.; Bauermeister, B.; Behrens, A.; Beltrame, P.; Bokeloh, K.; et al. Observation and applications of single-electron charge signals in the XENON100 experiment. J. Phys. 2014, G41, 035201. [CrossRef]

31. Akerib, D.S.; Alsum, S.; Araújo, H.M.; Bai, X.; Balajthy, J.; Baxter, A.; Bernard, E.P.; Bernstein, A.; Biesiadzinski, T.P.; Boulton, E.M.; et al. Investigation of background electron emission in the LUX detector. arXiv 2020, arXiv:2004.07791.

32. Sorensen, P.; Kamdin, K. Two distinct components of the delayed single electron noise in liquid xenon emission detectors. $J$. Instrum. 2018, 13, P02032. [CrossRef]

33. Sorensen, P. Electron train backgrounds in liquid xenon dark matter search detectors are indeed due to thermalization and trapping. arXiv 2017, arXiv:1702.04805.

34. Sato, K.; Yamashita, M.; Ichimura, K.; Itow, Y.; Kazama, S.; Moriyama, S.; Ozaki, K.; Suzuki, T.; Yamazaki, R. Development of Dual-phase Xenon TPC with a Quartz Chamber for Direct Dark Matter Search. arXiv 2019, arXiv:1910.13831.

35. Wei, Y.; Long, J.; Lombardi, F.; Jiang, Z.; Ye, J.; Ni, K. Development of a Sealed Liquid Xenon Time Projection Chamber with a Graphene-Coated Electrode. arXiv 2020, arXiv:2007.16194.

36. Xu, J.; Pereverzev, S.; Lenardo, B.; Kingston, J.; Naim, D.; Bernstein, A.; Kazkaz, K.; Tripathi, M. Electron extraction efficiency study for dual-phase xenon dark matter experiments. Phys. Rev. 2019, D99, 103024. [CrossRef]

37. Aprile, E.; Contreras, H.; Goetzke, L.W.; Melgarejo Fernandez, A.J.; Messina, M.; Naganoma, J.; Plante, G.; Rizzo, A.; Shagin, P.; Wall, R. Measurements of proportional scintillation and electron multiplication in liquid xenon using thin wires. J. Instrum. 2014, 9, P11012. [CrossRef]

38. Juyal, P.; Giboni, K.; Ji, X.; Liu, J. On Proportional Scintillation in Very Large LXe Detectors. Nucl. Sci. Tech. 2020, 31, 93. [CrossRef]

39. Lin, Q. Proposal of a Geiger-type Single-Phase Liquid Xenon Time Projection Chamber as Future Large Detector for Dark Matter Direct Search. arXiv 2021, arXiv:2102.06903.

40. Akimov, D.; Albert, J.; An, P.; Awe, C.; Barbeau, P.; Becker, B.; Belov, V.; Blackston, M.; Bolozdynya, A.; Brown, A.; et al. COHERENT 2018 at the Spallation Neutron Source. arXiv 2018, arXiv:1803.09183.

41. Miranda, O.G.; Papoulias, D.K.; Tórtola, M.; Valle, J.W.F. Probing neutrino transition magnetic moments with coherent elastic neutrino-nucleus scattering. J. High Energy Phys. 2019, 07, 103. [CrossRef]

42. Brudanin, V.B.; Medvedev, D.V.; Starostin, A.S.; Studenikin, A.I. New bounds on neutrino electric millicharge from GEMMA experiment on neutrino magnetic moment. Nucl. Part. Phys. Proc. 2016, 273-275, 2605-2608. [CrossRef]

43. Billard, J.; Johnston, J.; Kavanagh, B.J. Prospects for exploring New Physics in Coherent Elastic Neutrino-Nucleus Scattering. J. Cosmol. Astropart. Phys. 2018, 11, 16. [CrossRef]

44. Kopp, J.; Machado, P.A.; Maltoni, M.; Schwetz, T. Sterile neutrino oscillations: The global picture. J. High Energy Phys. 2013, 2013, 50. [CrossRef] 
45. Aprile, E.; Aalbers, J.; Agostini, F.; Alfonsi, M.; Althueser, L.; Amaro, F.D.; Antochi, V.C.; Angelino, E.; Angevaare, J.R.; Arneodo, F.; et al. Excess electronic recoil events in XENON1T. Phys. Rev. D 2020, 102, 072004. [CrossRef]

46. Fujikawa, K.; Shrock, R.E. Magnetic Moment of a Massive Neutrino and Neutrino-Spin Rotation. Phys. Rev. Lett. 1980, 45, 963-966. [CrossRef]

47. Bell, N.F.; Cirigliano, V.; Ramsey-Musolf, M.J.; Vogel, P.; Wise, M.B. How Magnetic is the Dirac Neutrino? Phys. Rev. Lett. 2005, 95, 151802. [CrossRef]

48. Bell, N.F.; Gorchtein, M.; Ramsey-Musolf, M.J.; Vogel, P.; Wang, P. Model independent bounds on magnetic moments of Majorana neutrinos. Phys. Lett. B 2006, 642, 377-383. [CrossRef]

49. Agostini, M.; Altenmüller, K.; Atroshchenko, V.; Bagdasarian, Z.; Basilico, D.; Bellini, G.; Benziger, J.; Bick, D.; Bonfini, G. Limiting neutrino magnetic moments with Borexino Phase-II solar neutrino data. Phys. Rev. D 2017, 96, 091103. [CrossRef]

50. Arceo-Diaz, S.; Schroder, K.P.; Zuber, K.; Jack, D. Constraint on the magnetic dipole moment of neutrinos by the tip-RGB luminosity in $\omega$-Centauri. Astropart. Phys. 2015, 70, 1-11. [CrossRef]

51. Córsico, A.; Althaus, L.; Bertolami, M.M.; Kepler, S.; García-Berro, E. Constraining the neutrino magnetic dipole moment from white dwarf pulsations. J. Cosmol. Astropart. Phys. 2014, 2014, 54. [CrossRef]

52. Beacom, J.F.; Vogel, P. Neutrino Magnetic Moments, Flavor Mixing, and the Super-Kamiokande Solar Data. Phys. Rev. Lett. 1999, 83, 5222-5225. [CrossRef]

53. Beda, A.; Brudanin, V.; Egorov, V.; Medvedev, D.; Pogosov, V.; Shevchik, E.; Shirchenko, M.; Starostin, A.; Zhitnikov, I. Gemma experiment: The results of neutrino magnetic moment search. Phys. Part. Nucl. Lett. 2013, 10, 139-143. [CrossRef]

54. De Gouvea, A.; Jenkins, J. What can we learn from neutrino electron scattering? Phys. Rev. D 2006, 74, 033004. [CrossRef]

55. Olive, K.A. Review of Particle Physics. Chin. Phys. C 2014, 38, 090001. [CrossRef]

56. Chen, J.W.; Chi, H.C.; Liu, C.P.; Wu, C.P. Low-energy electronic recoil in xenon detectors by solar neutrinos. Phys. Lett. B 2017, 774, 656-661. [CrossRef]

57. Aprile, E.; Aalbers, J.; Agostini, F.; Alfonsi, M.; Amaro, F.D.; Anthony, M.; Arneodo, F.; Barrow, P.; Baudis, L.; Bauermeister, B.; et al. XENON100 Dark Matter Results from a Combination of 477 Live Days. arXiv 2016, arXiv:1609.06154.

58. Szydagis, M.; Balajthy, J.; Brodsky, J.; Cutter, J.; Huang, J.; Kozlova, E.; Lenardo, B.; Manalaysay, A.; McKinsey, D.; Mooney, M.; et al. Noble Element Simulation Technique v2.0; Zenodo: Geneve, Switzerland, 2018. [CrossRef]

59. Vogel, P.; Engel, J. Neutrino Electromagnetic Form-Factors. Phys. Rev. D 1989, 39, 3378. [CrossRef]

60. Cowan, G.; Cranmer, K.; Gross, E.; Vitells, O. Asymptotic formulae for likelihood-based tests of new physics. Eur. Phys. J. C 2011, 71, 1-19. [CrossRef] 